\title{
The microwave spectrum, structure, and tunneling motion of the sulfur dioxide dimer
}

\author{
Amine Taleb-Bendiab, Kurt W. Hillig II, and Robert L. Kuczkowski \\ Department of Chemistry, University of Michigan, Ann Arbor, Michigan 48109-1055 USA
}

(Received 30 November 1990; accepted 11 February 1991)

\begin{abstract}
The microwave spectrum of $\left(\mathrm{SO}_{2}\right)_{2}$ has been reinvestigated using a pulsed beam Fouriertransform microwave spectrometer. Several new $a$-type transitions for the normal species and the $a$-type spectra of eight isotopically substituted species were measured. The spectra indicate that the $\mathrm{SO}_{2}$ dimer undergoes a high-barrier tunneling motion. Based on the analysis used for $\left(\mathrm{H}_{2} \mathrm{O}\right)_{2}$ by Coudert and Hougen [J. Mol. Spectrosc. 130, 86 (1988)], the internal motion is identified as a geared interconversion motion similar to that displayed by $\left(\mathrm{H}_{2} \mathrm{O}\right)_{2}$. From the analysis of the moments of inertia of the various isotopic species, an ac plane of symmetry is established for the dimer and the tilt angles of the $C_{2}$ axes of each subunit relative to the line joining their centers of mass were determined. From Stark effect measurements, $\mu_{a}$ was redetermined and $\mu_{c}$ was shown to be nearly zero. Electrostatic calculations using distributed multipoles were carried out to explore the structure of this dimer.
\end{abstract}

\section{INTRODUCTION}

The gas-phase complexes involving mixtures of species such as $\mathrm{H}_{2} \mathrm{O}, \mathrm{O}_{3}$, and $\mathrm{SO}_{2}$ are of great interest in the atmospheric sciences. One of these complexes, $\left(\mathrm{SO}_{2}\right)_{2}$, may be important in the "acid rain" phenomenon as discussed by Nelson, Fraser, and Klemperer in a paper reporting its radio frequency and microwave spectrum. ${ }^{1}$ The spectrum was characteristic of an asymmetric top in which the two monomer units undergo a high-barrier interconversion motion. The motion was discerned from small shifts in the $a$-type transitions consistent with a tunneling splitting of $70 \mathrm{kHz}$. Since $\mu_{a}$ was antisymmetric with respect to the tunneling process, a motion involving the interchange of two nonequivalent $\mathrm{SO}_{2}$ subunits was proposed. However, the structure of the complex could not be established unambiguously precluding a more detailed description of the tunneling motion. The distance $R_{\mathrm{cm}}$ between the centers of mass of the two subunits was established as $3.825 \AA$. The dipole component $\mu_{a}$ was $1.4052 \mathrm{D}$. The assigned $a$-type transitions for the $\mathrm{SO}_{2}$ dimer were recently extended by Matsumura, Lovas, and Suenram. ${ }^{2}$

While we were studying the microwave spectrum of benzene- $\mathrm{SO}_{2}$ and its isotopic species, ${ }^{3}$ we encountered transitions which did not belong to this complex. Based on the previous studies of $\left(\mathrm{SO}_{2}\right)_{2}$, we have been able to assign many of these transitions to the normal species of this dimer and various ${ }^{18} \mathrm{O}$ and ${ }^{34} \mathrm{~S}$ isotopic species. These new data have allowed us to determine the equilibrium orientation of the two $\mathrm{SO}_{2}$ monomers. Knowledge of the structure enabled us to analyze the frameworks between which the dimer may tunnel. A single interconversion tunneling motion can account for the tunneling effects observed in the spectra.

\section{EXPERIMENTAL}

The microwave spectrum of $\left(\mathrm{SO}_{2}\right)_{2}$ was measured in a Balle-Flygare Fourier-transform microwave (FTMW) spectrometer with a pulsed nozzle source. ${ }^{4}$ A mixture of about $1 \% \mathrm{SO}_{2}$ and $99 \% \mathrm{Ne}$ at a pressure of 1 to 2 atm in $1 \mathrm{~L}$ glass storage vessels was used. Line widths full width at half maximum (FWHM) were 15 to $30 \mathrm{kHz}$ and the frequency measurement accuracy was estimated to be $4 \mathrm{kHz}$, except for the double- ${ }^{18} \mathrm{O}$ isotopically substituted species, where transitions occurred as doublets, some of which were partially resolved. For these isotopic species, conventional resolution enhancement signal processing techniques were used and the accuracy of the frequency measurements was estimated to be $10 \mathrm{kHz}$.

The spectrometer was equipped with Stark plates for the measurement of Stark effects. ${ }^{5}$ The $2_{02} \leftarrow 1_{11}$ transition of $\mathrm{SO}_{2}$ was used as an electric field calibration standard $\left[\mu\left(\mathrm{SO}_{2}\right)\right]=1.63305 \mathrm{D}^{6}{ }^{6}$ Stark shifts were measured at electric field values between 0 and $500 \mathrm{~V} / \mathrm{cm}$.

The spectra of the ${ }^{34} \mathrm{SO}_{2}-\mathrm{SO}_{2}$ and $\mathrm{SO}_{2}-{ }^{34} \mathrm{SO}_{2}$ species were observed in natural abundance $\left({ }^{34} \mathrm{~S}, 4 \%\right) \cdot \mathrm{S}^{18} \mathrm{O}_{2}(99 \%$ enrichment, Alfa Products) was used without dilution to assign the $\left(\mathrm{S}^{18} \mathrm{O}_{2}\right)_{2}$ spectrum. The spectra of the single- ${ }^{18} \mathrm{O}$ and double- ${ }^{18} \mathrm{O}$ species were observed starting with a 50\%$50 \%$ mixture of $\mathrm{S}^{16} \mathrm{O}_{2}$ and $\mathrm{S}^{18} \mathrm{O}_{2}$, which rapidly exchanged to form a statistical 2:1:1 mixlure of $\mathrm{S}^{16} \mathrm{O}^{18} \mathrm{O}: \mathrm{S}^{16} \mathrm{O}_{2}: \mathrm{S}^{18} \mathrm{O}_{2}$.

\section{RESULTS AND ANALYSIS}

\section{A. Spectral assignments}

\section{1. $\left(\mathrm{SO}_{2}\right)_{2}$ and $\left(\mathrm{S}^{18} \mathrm{O}_{2}\right)_{2}$}

The rotational spectrum of $\left(\mathrm{SO}_{2}\right)_{2}$ was first studied by Nelson et al. ${ }^{\prime}$ The transitions arose from states with $K_{p} \geqslant 2$ for the $Q$ branch and $K_{p} \geqslant 3$ for the $R$ branch. More recently, eighteen $R$-branch transitions associated with the $K_{p}=0,1$, and 2 states were measured by Matsumura et al. ${ }^{2}$ We have measured eleven additional $R$-branch transitions $(J=4 \leftarrow 3$, $5 \leftarrow 4$, and $6 \leftarrow 5$ ). The complete set of assigned transitions is shown in Table $\mathrm{I}$.

$\left(\mathrm{SO}_{2}\right)_{2}$ has a plane of symmetry (see Sec. III C). Analogous to $\left(\mathrm{H}_{2} \mathrm{O}\right)_{2},{ }^{7}$ there are eight possible equivalent 
TABLE I. Rotational transitions of $\left(\mathrm{SO}_{2}\right)_{2}$.

\begin{tabular}{|c|c|c|c|c|c|c|c|}
\hline \multirow[b]{2}{*}{$J^{\prime} A_{n} \Lambda_{. .}$} & \multirow[b]{2}{*}{ 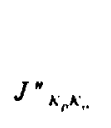 } & \multicolumn{2}{|c|}{$A_{1}^{ \pm}$state } & \multirow[b]{2}{*}{$J^{\prime}{ }_{k, k_{1}}$} & \multirow[b]{2}{*}{$J^{\prime \prime}{ }_{\kappa_{p}, K_{,}}$} & \multicolumn{2}{|c|}{$A_{I^{\circ}}$ state } \\
\hline & & $v_{s, b m}(M H z)$ & $\Delta v(\mathrm{kHz})^{a}$ & & & $v_{\text {thte. }}(\mathrm{MHz})$ & $\Delta v(\mathrm{kHz})^{\mathrm{a}}$ \\
\hline 220 & 221 & $0.175^{b}$ & -3 & $6_{06}$ & $5_{05}$ & $11103.176^{\circ}$ & -1 \\
\hline 321 & $3_{22}$ & $1.308^{b}$ & -1 & $6_{16}$ & $5_{15}$ & $10974.964^{\circ}$ & 1 \\
\hline $4_{22}$ & $4_{2,3}$ & $3.648^{\mathrm{h}}$ & 0 & $6_{15}$ & $5_{14}$ & $11242.620^{\circ}$ & 0 \\
\hline $5_{21}$ & $5_{24}$ & $8.743^{\mathrm{b}}$ & -0 & $6_{2 s}$ & $5_{24}$ & 11106.208 & -0 \\
\hline $6_{24}$ & $6_{3 s}$ & $17.276^{b}$ & 1 & $6_{24}$ & $5_{23}$ & 11114.740 & -0 \\
\hline $7: 3$ & $7_{26}$ & $31.282^{\mathrm{b}}$ & 1 & $6_{34}$ & $5_{33}$ & $11102.715^{\mathrm{b}}$ & -1 \\
\hline 927 & $9_{28}$ & $81.700^{t}$ & -2 & 633 & $5_{32}$ & $11102.626^{b}$ & 1 \\
\hline 73 & $7_{35}$ & $0.249^{b}$ & 1 & $6_{43}$ & $5_{42}$ & $11093.859^{6}$ & -4 \\
\hline $8: 5$ & 8,8 & $0.325^{b}$ & 1 & 642 & $5+1$ & $11093.724^{\mathrm{b}}$ & 1 \\
\hline 4,3 & $4_{14}$ & $446.341^{b}$ & -1 & $7_{07}$ & $6_{\nu_{0}}$ & $12949.332^{\circ}$ & 1 \\
\hline 5,4 & $5_{15}$ & $669.670^{\mathrm{b}}$ & 0 & 717 & $6_{b a}$ & $12802.296^{\circ}$ & 1 \\
\hline 6,5 & 6, & $937.325^{13}$ & -2 & $7_{10}$ & 6,15 & $13114.809^{\circ}$ & 0 \\
\hline 716 & 717 & $1249.842^{b}$ & 2 & $7_{20}$ & $6_{2 s}$ & $12955.707^{\circ}$ & 0 \\
\hline $4_{144}$ & 30.3 & 7406.077 & -3 & $7_{25}$ & 624 & $12969.713^{c}$ & 1 \\
\hline $4_{14}$ & 318 & 7318.148 & 0 & $7_{35}$ & $6_{34}$ & $12952.687^{b}$ & -1 \\
\hline 4,1 & $3 / 2$ & 7496.568 & -1 & $7_{34}$ & 6,3 & $12952.936^{\mathrm{h}}$ & 2 \\
\hline $4_{23}$ & $3_{22}$ & 7405.382 & 2 & $7_{44}$ & 64 & $12942.151^{\mathrm{b}}$ & 6 \\
\hline $4_{22}$ & 321 & 7407.718 & -1 & $7_{43}$ & $6_{42}$ & $12942.281^{b}$ & -4 \\
\hline 4,2 & $3_{31}$ & 7402.112 & -4 & 8 & $7_{07}$ & $14793.128^{\circ}$ & -1 \\
\hline 43 & $3_{w}$ & 7401.976 & -5 & $8_{18}$ & $7_{17}$ & $14629.148^{\mathrm{c}}$ & 4 \\
\hline $5_{t s}$ & $4_{04}$ & $9255.516^{\circ}$ & 1 & 817 & 7,6 & $14985.907^{\circ}$ & 1 \\
\hline$S_{15}$ & $4_{14}$ & 9146.690 & -2 & 909 & $8_{08}$ & $16634.846^{\circ}$ & -1 \\
\hline $5_{14}$ & 4,3 & 9370.020 & 1 & 9 & 8 & $16454.848^{\circ}$ & -1 \\
\hline $5_{24}$ & $4_{23}$ & $9255.884^{\mathrm{e}}$ & 1 & $9_{18}$ & 8,7 & $16856.344^{\circ}$ & -1 \\
\hline $5_{23}$ & $4_{22}$ & $9260.980^{\circ}$ & 1 & $10_{24}$ & $9_{28}$ & $18500.550^{\circ}$ & -3 \\
\hline $5_{3}$ & $4_{32}$ & $9252.336^{\mathrm{b}}$ & 5 & & & & \\
\hline $5_{32}$ & $4_{31}$ & $9252.488^{\circ}$ & -0 & & & & \\
\hline $5_{42}$ & $4+1$ & $9245.088^{\circ}$ & 2 & & & & \\
\hline $5_{41}$ & $4_{40}$ & $9245.225^{\mathrm{h}}$ & -1 & & & & \\
\hline
\end{tabular}

" $\Delta v=v_{\text {situ }}-v_{\text {collic }}$ using the constants in Table III.

'Taken from Ref. 1.

'Taken from Ref. 2.

frameworks among which $\left(\mathrm{SO}_{2}\right)_{2}$ may have feasible tunneling motions. These frameworks are shown in Fig. 1, where the equilibrium configuration from the results in Sec. III C is used. In parallel with Hougen and Coudert's theoretical papers on $\left(\mathrm{H}_{2} \mathrm{O}\right)_{2}, 8,9$ the framework numbering in Fig. 1 has been kept identical to Ref. 8 , although the atom numbers were not kept the same. The possible tunneling motions are: $1 \rightarrow 2,1 \rightarrow 3,1 \rightarrow 4,1 \rightarrow 5$ (or $1 \rightarrow 6$ ), and $1 \rightarrow 7$ (or $1 \rightarrow 8$ ). Other framework pairing combinations are related by symmetry. Unlike $\left(\mathrm{H}_{2} \mathrm{O}\right)_{2}$, the tunneling motion $1 \rightarrow 4$ in $\left(\mathrm{SO}_{2}\right)_{2}$ is very probably unfeasible due to steric effects, in other words, it has a very high barrier. On the same grounds, the $1 \rightarrow 7$ motion which corresponds to an antigeared motion can be ruled out. However, the $1 \rightarrow 3$ motion might have a lower barrier than the $1 \rightarrow 4$ motion. The $1 \rightarrow 3$ and $1 \rightarrow 4$ motions can be viewed as internal rotations (some other pathways might also be possible) of the in-plane and the out-of-plane monomers, respectively, about their $C_{2}$ axes. The $1 \rightarrow 2$ motion can be formally described as a geared rotation of both monomers about their respective $C_{2}$ axes or about axes perpendicular to the plane of symmetry of the dimer and may have a lower barrier than the $1 \rightarrow 4$ and $1 \rightarrow 7$ motions. The $1 \rightarrow 5$ motion might also have a low enough barrier to be

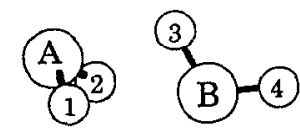

2
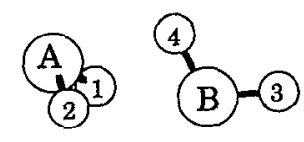

6
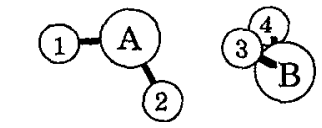

3
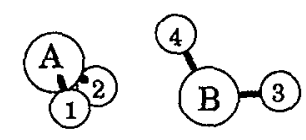

7
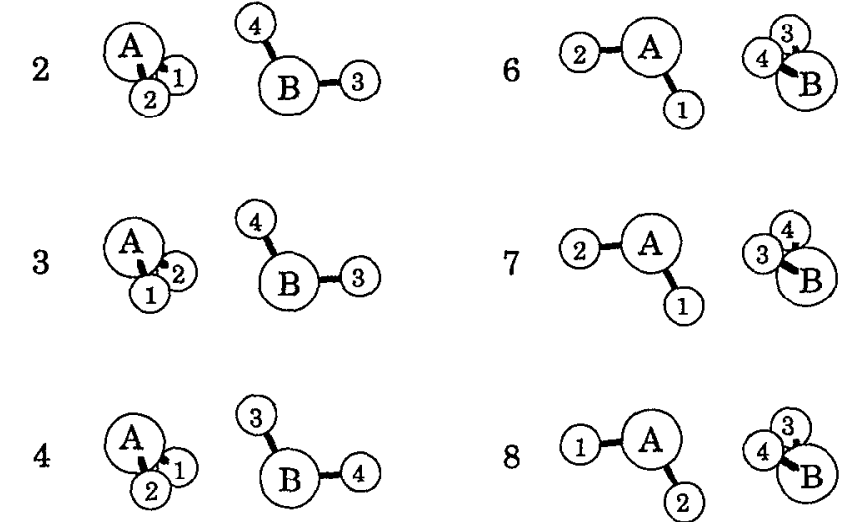

8
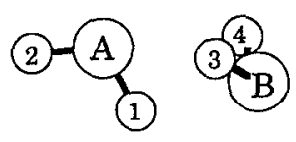

FIG. 1. Numbering of the eight equivalent frameworks of $\left(\mathrm{SO}_{2}\right)_{2}$. The frameworks are viewed along the $b$ axis of the dimer. The circled numbers 1 to 4 indicate the four oxygens and the circled letters $A$ and $B$ indicate the two sulfurs. 
detected and must, indeed, be the interconversion motion first identified by Nelson et al. ${ }^{\prime}$ This tunneling, analogous to the geared interconversion motion in $\left(\mathrm{H}_{2} \mathrm{O}\right)_{2}{ }^{9}$ or $(\mathrm{HF})_{2}{ }^{10}$ is a $90^{\circ}$ geared rotation of each monomer about its $C_{2}$ axis followed by a simultaneous readjustment of the tilt angles of these axes relative to the center-of-mass distance. As in $\left(\mathrm{H}_{2} \mathrm{O}\right)_{2},{ }^{9}$ the $1 \rightarrow 5$ interconversion motion splits each rotational state into three substates, where one is doubly degenerate. If the $1 \rightarrow 3$ or $1 \rightarrow 2$ motion also occurs, i.e., has a low enough barrier to cause a splitting, then each of the three substates will further split into two other substates giving six levels. Since the ${ }^{16} \mathrm{O}$ nucleus has a spin of zero, only one sublevel among the maximum of six has a statistical weight different from zero. This sublevel has the symmetry $A_{i}{ }_{1}$ in the $G_{16}$ permutation-inversion group. Therefore, the spectrum should display one series of transitions and moreover the $1 \rightarrow 2$ or $1 \rightarrow 3$ motion, even if they cause a splitting, cannot be detected and only the $1 \rightarrow 5$ motion is observed. This can be verified by examining the effect of each motion on the eigenvalues as summarized for the $\mathrm{H}_{2} \mathrm{O}$ dimer in Table IV of Ref. 9(a).

The Hamiltonian applied to fit the $A_{1}^{ \pm}$transitions of $\left(\mathrm{SO}_{2}\right)_{2}$ is

$$
\mathscr{H}=\mathscr{H}^{\circ}+\alpha(-1)^{J+K}\left(2 \times h_{S^{\prime \prime}}\right),
$$

where $\mathscr{H}^{\circ}$ is a semirigid-rotor Hamiltonian, i.e., the usual rigid-rotor Hamiltonian (using the $I^{r}$ representation) to which the Watson $S$-reduction centrifugal distortion Hamiltonian $^{11}$ is added. The second term in Eq. (1) arises from the $1 \rightarrow 5$ tunneling motion, where the notation introduced by Coudert and Hougen ${ }^{9}$ has been used. The phase factor is identical to that of the $1 \rightarrow 5$ motion in the $\mathrm{H}_{2} \mathrm{O}$ dimer; ${ }^{9(b)}$ for $K=0, \alpha=1$, and for $K>0, \alpha=(-1)^{j+K}$ for $A^{\prime}$ and $-(-1)^{J+K}$ for $A^{\prime \prime}$ rotational wave functions in the $C_{b}$ group of the equilibrium configuration of the dimer. Since $\left(\mathrm{SO}_{2}\right)_{2}$ is a relatively heavy complex, no higher distortion terms associated with the $1 \rightarrow 5$ motion which are $J$ and $K$ dependent were necessary. By comparing the second term in the Hamiltonian used by Nelson et al. ${ }^{1}$ and in the present work, we notice that $h_{5 v}=-\delta_{\text {inv }} / 4$.

Because $\left(\mathrm{S}^{18} \mathrm{O}_{2}\right)_{2}$ has the same symmetry as the $\mathrm{SO}_{2}$ dimer normal species and ${ }^{18} \mathrm{O}$ also has a nuclear spin of zero, the above discussion systematically applies to $\left(\mathrm{S}^{18} \mathrm{O}_{2}\right)_{2}$. The spectral fit for $\left(\mathrm{SO}_{2}\right)_{2}$ is shown in Table I. The fit for a selected set of transitions of $\left(\mathrm{S}^{18} \mathrm{O}_{2}\right)_{2}$ is given in Table II; The complete set of transitions for this isotope and all subsequent isotopes is available as supplementary malerial. ${ }^{12}$ The derived spectroscopic constants for both species are listed in Table III.

Based on the structure of $\left(\mathrm{SO}_{2}\right)_{2}$ (see below), the spectrum of this dimer should be characterized by $a$-type and $c$ type selection rules. However, only $a$-type transitions have been observed. We have searched for the $c$-type $1_{10} \leftarrow 0_{\infty 0}$

TABLE II. Selected rotational transitions for isotopic species of the SO, dimer.

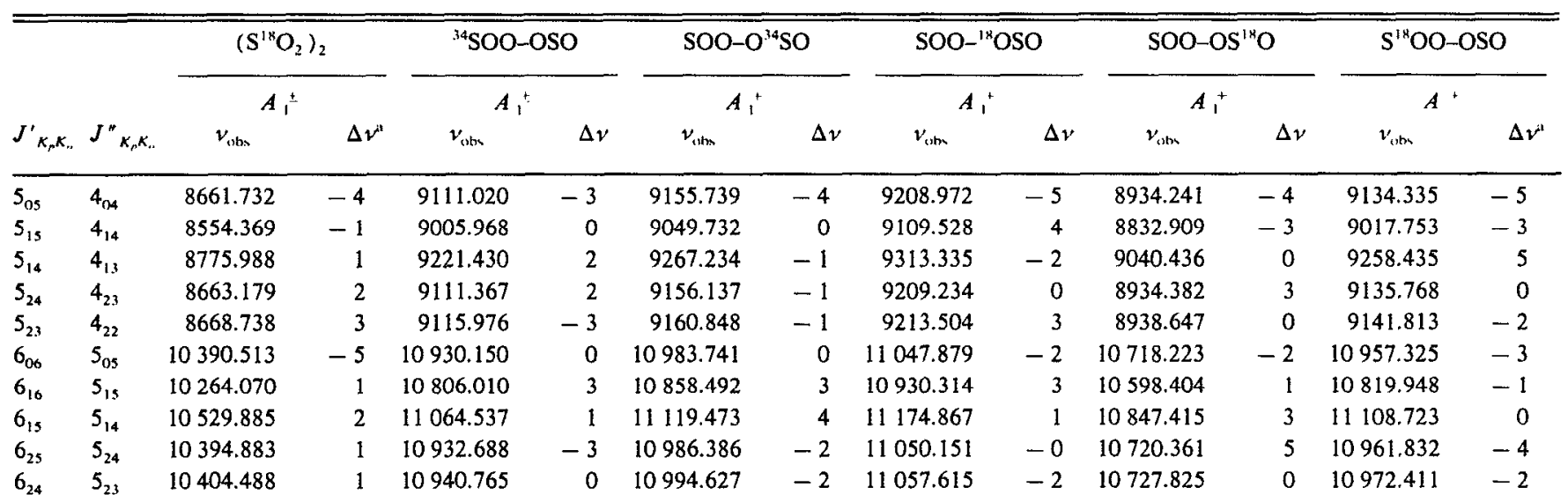

\begin{tabular}{|c|c|c|c|c|c|c|c|c|c|c|}
\hline & \multicolumn{4}{|c|}{$\mathrm{S}^{18} \mathrm{OO}^{18}{ }^{18} \mathrm{OSO}$} & \multicolumn{4}{|c|}{$\mathrm{S}^{18} \mathrm{OO}-\mathrm{OS}^{1 \times} \mathrm{O}$} & \\
\hline & & \multicolumn{2}{|c|}{$A^{ \pm}$} & \multicolumn{2}{|c|}{$A^{\mp}$} & \multicolumn{2}{|c|}{$A \pm$} & \multicolumn{2}{|c|}{$A^{\top}$} & \\
\hline & & $\nu_{\mathrm{sbm}}$ & $\Delta v$ & $v_{\mathrm{obs}}$ & $\Delta v$ & $v_{\text {utu }}$ & $\Delta v$ & $v_{\text {stits }}$ & $\Delta v$ & \\
\hline 5,5 & $4_{14}$ & 8979.525 & 0 & 8979.582 & -1 & 8707.673 & -1 & 8707.704 & -3 & \\
\hline $5_{14}$ & $4_{13}$ & 9201.060 & 0 & 9201.006 & 3 & 8931.486 & -1 & 8931.459 & 5 & \\
\hline $5_{24}$ & $4_{23}$ & 9088.140 & 9 & 9088.195 & 7 & 8817.341 & 5 & 8817.369 & 0 & \\
\hline $66_{15}$ & $5_{14}$ & 11039.940 & 2 & 11039.995 & -1 & 10716.514 & -2 & 10716.549 & 0 & \\
\hline $6_{25}$ & $S_{24}$ & 10904.815 & 1 & 10904.757 & 1 & 10579.869 & -3 & 10579.837 & -2 & \\
\hline $6_{24}$ & $5_{23}$ & 10913.993 & 1 & 10914.050 & 1 & 10588.936 & -4 & 10588.975 & 2 & \\
\hline
\end{tabular}

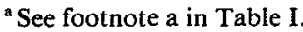


TABLE III. Spectroscopic constants for isotopic species of the $\mathrm{SO}_{2}$ dimer.

\begin{tabular}{|c|c|c|c|c|c|c|c|c|c|}
\hline & $(50,)^{\prime \prime}$ & $\left(\mathrm{S}^{\prime} \mathrm{O}_{2}\right)_{2}$ & "SOO-USO & $500-0^{2}+50$ & sou-' "OSO & $500-05^{14} \mathrm{O}$ & $\mathrm{S}^{\prime+} \mathrm{OO}-\mathrm{OSO}$ & $5^{14} \mathrm{OO}^{-14} \mathrm{OSO}$ & $S^{14} 00-0 S^{1 x} \mathrm{O}$ \\
\hline$A(M H z)$ & $6923.5(5)^{n}$ & $6186.1(15)$ & $6917.2(35)$ & $6916.8(39)$ & $6749.4(31)$ & $6918.9(28)$ & $6628.3(27)$ & $6462.9(12)$ & $6621.3(25)$ \\
\hline$B(\mathrm{MHz})$ & $948.4678(1)$ & $888.9752(4)$ & $933.2138(4)$ & $937.9036(4)$ & $941.8105(4)$ & $914.7041(5)$ & $938.2142(5)$ & $931.4851(3)$ & $904.6403(4)$ \\
\hline$C\left(\mathrm{MH}_{2}\right)$ & $903.8250(1)$ & $844.6558(4)$ & $890.1186(5)$ & $894.3995(5)$ & $901.0449(4)$ & $873.1963(5)$ & $890.0744(6)$ & $887.1855(3)$ & $859.8804(4)$ \\
\hline$D_{1}(\mathrm{kHz})$ & $2.0741(5)$ & $1.802(4)$ & $1.999(5)$ & $2.082(5)$ & $2.044(4)$ & $1.934(4)$ & $2.038(5)$ & $1.991(3)$ & $1.881(7)$ \\
\hline$D_{j k}(\mathrm{kHz})$ & $97.92(3)$ & $86.29(9)$ & $94.85(10)$ & $95.52(11)$ & $88.77(10)$ & $92.26(11)$ & $99.95(13)$ & $90.50(7)$ & $93.65(11)$ \\
\hline$d_{:}(\mathrm{kHz})$ & $-0.0299(5)$ & $-0.0246(22)$ & $-0.0259(34)$ & $-0.0223(39)$ & $-0.0109(29)$ & $-0.0226(29)$ & $-0.0388(46)$ & $-0.0228(19)$ & $-0.0361(34)$ \\
\hline$h_{1}(\mathrm{hHz})$ & $-17.43(8)$ & $-5.32(23)$ & & & & & & $-14.38(36)$ & $-8.25(39)$ \\
\hline$n$ & 54 & 16 & 14 & 14 & 20 & 15 & 17 & 32 & 20 \\
\hline$d_{1} \ldots . ., k(k H z)$ & 2 & 3 & 3 & 3 & 4 & 4 & 5 & 4 & 4 \\
\hline
\end{tabular}

"Additional centrifugal distortion constants $(\mathrm{kHz})$ determined for this species were $d_{1}=-0.0052(6), H_{J K}=-0.0071(2), H_{\kappa J}=-0.0407(16)$.

"The uncertainties are $1 \sigma$.

'Number of transitions in the fit.

$\Delta v=v_{i, m}-v^{\prime}$

transition within $\pm 10 \mathrm{MHz}$ of the prediction from the fit of the $a$-type transitions without observing any candidates. A more extensive search was not undertaken since $\mu_{\mathrm{c}} \approx 0$ (see Sec. III B).

\section{2. ${ }^{34} \mathrm{SOO-OSO}$ and $500-\mathrm{O}^{34} \mathrm{SO}$}

The two single $-{ }^{34} \mathrm{~S}$ species have only four equivalent frameworks $1,2,3$, and 4 . Since the $1 \rightarrow 5$ motion is absent in this case, the spectra will not ordinarily exhibit any effects from this tunneling process. It is possible for the $1 \rightarrow 5 \mathrm{mo}$ tion to be unquenched ${ }^{9(a)}$ if the tunneling splitting is very large compared to the difference of the zero-point energy between the two isotopic species. This phenomenon was observed in the mixed ${ }^{35} \mathrm{Cl}-{ }^{37} \mathrm{Cl}$ isotopic species of $(\mathrm{HCl})_{2} \cdot{ }^{13}$ Not surprisingly, this effect has not been observed between ${ }^{34} \mathrm{SOO}-\mathrm{OSO}$ and $\mathrm{SOO}-\mathrm{O}^{34} \mathrm{SO}$ presumably because the ratio of the zero-point energy difference to the interconversion splitting is large. From symmetry considerations, and whether or not there is any tunneling splitting from the $1 \rightarrow 2$, 3,4 motions, only one state of symmetry $A_{1}^{ \pm}$has a nonzero statistical weight. The group and symmetry labels used are analogous to those employed for $\mathrm{H}_{2} \mathrm{O}-\mathrm{DOD} .^{9(\mathrm{a})}$ Again, no evidence of splitting for any of the motions will show up [see Table IX in Ref. 9(a) ]. (Note: The effect of the $1 \rightarrow 3$ motion is not given in Table IX; It should occur as $+\mathrm{H}_{13}$ in the expressions for $A_{1}{ }^{+}$.) Selected rotational transitions are listed in Table II and the derived spectroscopic constants using the regular semirigid-rotor Hamiltonian $\left(\mathscr{H}^{\circ}\right)$ are reported in Table III.

\section{3. $S O O-{ }^{18} \mathrm{OSO}, \mathrm{SOO}-0 \mathrm{~S}^{18} \mathrm{O}$, and $\mathrm{S}^{18} \mathrm{OO}$-OSO}

The two single $-{ }^{18} \mathrm{O}$ species, SOO- ${ }^{18} \mathrm{OSO}$ and SOO$\mathrm{OS}^{1 \mathrm{I}} \mathrm{O}$, have two equivalent frameworks: 1 and 4 . Even if there is splitting from the $1 \rightarrow 4$ tunneling motion, only one substate of symmetry $A_{ \pm}^{ \pm}$in the same group used for $\mathrm{H}_{2} \mathrm{O}$ $\mathrm{DOH}^{9(\mathrm{a})}$ has a statistical weight different from zero and evidence for the splitting can not be observed from the transitions [ see Table VII in Ref. 9(a) ]. Consequently, the spectra of these two isotopic species are fit by a semirigid-rotor Hamiltonian $\left(\mathscr{H}^{\circ}\right)$. Selected rotational transitions are listed in Table II for SOO- ${ }^{18} \mathrm{OSO}$ and SOO-OS ${ }^{18} \mathrm{O}$ and the spectroscopic constants are given in Table III.

For $\mathrm{S}^{18} \mathrm{OO}-\mathrm{OSO}$, the plane of symmetry in its equilibrium configuration is absent and four equivalent frameworks exist: 1, 2, 3 and 4. Analogous to the situation for HDODOD, ${ }^{9(a)}$ the $1 \rightarrow 4$ and $1 \rightarrow 2$ motions will split each level into four components with symmetries $A \pm$ and $B \pm$. Only the $A \pm$ levels will have a statistical weight of one and doublets may arise. Nevertheless, transitions arising from only one state have been found. Two explanations are possible: Either the upper state is very high in energy relative to the lower state and, therefore, it cannot be populated due to the low temperature of the pulsed beam $(\sim 2 \mathrm{~K})$ as occurred for example in HDO-DOD ${ }^{14}$ or the barrier height for the $1 \rightarrow 4$ and $1 \rightarrow 2$ motions are too high for this isotopic species to display a resolvable splitting. We are more inclined to believe the second possibility because of the mass of the $\mathrm{SO}_{2}$ subunits and as discussed before, at least the $1 \rightarrow 4$ motion should have a very high barrier. Also, the $1 \rightarrow 3$ motion does not add any splitting effects to the transitions due to nuclear spin restrictions. Selected transitions of $\mathrm{S}^{18} \mathrm{OO}-\mathrm{OSO}$ are given in Table II where the semirigid-rotor Hamiltonian $\left(\mathscr{H}^{\circ}\right)$ was used. The derived spectroscopic constants are shown in Table III.

\section{4. $\mathrm{S}^{18} \mathrm{OO}-{ }^{18} \mathrm{OSO}$ and $\mathrm{S}^{18} \mathrm{OO}-\mathrm{OS}^{18} \mathrm{O}$}

For $\mathrm{S}^{18} \mathrm{OO}-{ }^{18} \mathrm{OSO}$ and $\mathrm{S}^{18} \mathrm{OO}-\mathrm{OS}{ }^{18} \mathrm{O}$, the frameworks are $1,4,5$, and 8 or $1,4,6$, and 7 . Here again, the $1 \rightarrow 5$ (or $1 \rightarrow 6$ ) tunneling motion appears. If we assume as before that the tunneling splitting effects of the $1 \rightarrow 4$ and $1 \rightarrow 8$ (or $1 \rightarrow 7$ ) motions are small, the geared interconversion motion splits each rotational state into two sublevels with symmetry spe$\operatorname{cies} A^{-}$and $A^{+}$and equal spin weights in the group uscd to describe HDO-DOH. ${ }^{9(a)}$ The nature of the splitting is such that doublets are expected for these species [see, for example, Fig. 4 in Ref. 9(a)] and this was observed.

The Hamiltonian applied to fit the $\mathrm{A}^{\ddagger}$ transitions of $\mathrm{S}^{18} \mathrm{OO}-{ }^{18} \mathrm{OSO}$ and $\mathrm{S}^{18} \mathrm{OO}-\mathrm{OS}^{18} \mathrm{O}$ is

$$
\mathscr{H}=\mathscr{H}^{\circ} \pm h_{\mathrm{s}}
$$

This Hamiltonian is similar to that defined in Eq. (1), except 
that the factor of 2 in the second term has been dropped. This is due to the absence of the companion $1 \rightarrow 6$ (or $1 \rightarrow 5$ ) motion. Also, the phase factor quantities dependent on $J, K$, and on the $A^{\prime}, A^{\prime \prime}$ rotational symmetry have been dropped due to the absence of $C_{s}$ symmetry. Selected transitions of these two species are shown in Table II and their corresponding spectroscopic constants are given in Table III. It is interesting to note that the magnitude of the splitting parameter $\left|h_{5 v}\right|$ decreases from the normal to the double ${ }^{18} \mathrm{O}$ to the tetra- ${ }^{18} \mathrm{O}$ species.

\section{B. Dipole moment}

The Stark shifts for nine $M$ components from five transitions were measured. The second-order Stark coefficients were obtained from fits of $\Delta v$ vs $\mathscr{C}^{2}$. The observed coefficients arc listed in Table IV. Plots of $\Delta v / \mathscr{E}^{2}$ vs $\mathscr{E}^{2}$ showed no significant curvature from nonsecond-order effects. A leastsquares fit of the observed coefficients yielded $\mu_{a}=1.408$ (1) D. This is nearly identical to the value of 1.4052(10) D determined by Nelson et al. ${ }^{1}$ from two $Q$ branch transitions. The fit also gave $\mu_{c}^{2}=-0.0017 \mathrm{D}^{2}$, suggesting that $\mu_{c}$ is too small to be determined from the Stark shifts of the selected transitions. An upper limit of $0.08 \mathrm{D}$ was estimated for $\mu_{c}$ by assuming that it contributes a shift of $10 \mathrm{kHz}$ to the $5_{05} \leftarrow 4_{04}, M=4$ transition at a field of 440 $\mathrm{V} / \mathrm{cm}$. The $\mu_{b}$ component was not included in the fit because of the existence of an $a c$ plane of symmetry in the complex, which will be discussed in the following section.

\section{Structure}

The planar second moment of inertia of $\left(\mathrm{SO}_{2}\right)_{2}$, $P_{b b}=1 / 2\left(I_{c}+I_{a}-I_{b}\right)=\Sigma m_{i} b_{i}^{2}=49.66 \mathrm{amu} \times \AA^{2}$, is very close to $P_{a a}=49.05 \mathrm{amu} \times \AA^{2}$ for free $\mathrm{SO}_{2} .{ }^{15}$ This suggests that the $\mathrm{SO}_{2}$ dimer has an ac plane of symmetry where one monomer lies in the plane of symmetry while the two oxygens for the second monomer straddle it. Moreover, the observation that $P_{c c}$ is 23.34 amu $\times \AA^{2}$, clearly eliminates the possibility that the dimer is planar.

Two singly substituted ${ }^{34} \mathrm{~S}$ species were observed confirming that there are two structurally nonequivalent $\mathrm{SO}_{2}$

TABLE IV. Stark coefficients and dipole components for $\left(\mathrm{SO}_{2}\right)_{2}$.

\begin{tabular}{lccc}
\hline \hline Transition & $|\mathrm{M}|$ & $\Delta v / \mathscr{C}^{2 \mathrm{a}}$ & obs-calc $^{\mathrm{b}}$ \\
\hline $5_{03}-4_{04}$ & 4 & 0.354 & 0.001 \\
$5_{14}-4_{13}$ & 3 & -1.642 & -0.005 \\
& 4 & -2.838 & 0.002 \\
$6_{06}-5_{05}$ & 5 & 0.217 & -0.000 \\
$6_{16}-5_{15}$ & 4 & 0.965 & 0.001 \\
& 5 & 1.537 & -0.000 \\
$6_{15}-5_{14}$ & 3 & -0.438 & 0.000 \\
& 4 & -0.736 & -0.001 \\
& 5 & -1.112 & 0.003 \\
& $\left|\mu_{\mathrm{a}}\right|=1.408(1)^{\mathrm{c}} \mathrm{D}$ & $\left|\mu_{\mathrm{c}}\right|<0.08 \mathrm{D}$ \\
\hline
\end{tabular}

\footnotetext{
Observed Stark coefficients in units of $10^{-5} \mathrm{MHz} /(\mathrm{V} / \mathrm{cm})^{2}$.

${ }^{\text {b }}$ Stark coefficients calculated using rotational constants in Table III.

"The uncertainty is $2 \sigma$.
}

species in the dimer. Each specics has a $P_{b b}$ value of 49.64 amu $\times \AA^{2}$. This implies that the two sulfur atoms lie in the $a c$ plane of symmetry. The single- ${ }^{18} \mathrm{O}$ substituted species lead to $P_{b b}$ values of $52.69,49.58$, and $49.65 \mathrm{amu} \times \AA^{2}$. The first value is much larger than the $P_{b b}$ of the normal species and similar to the value $P_{a a}=52.04 \mathrm{amu} \times \AA^{2}$ for $\mathrm{S}^{18} \mathrm{O}^{16} \mathrm{O}^{16}$ indicating that the substituted oxygen in this isotopomer lies out of the plane of symmetry. For the other two isotopomers, the value of $P_{b b}$ has changed little compared to the normal species, indicating that the substituted oxygen atoms lie in the plane of symmetry. These observations suggest that the orientation of the two subunits in $\left(\mathrm{SO}_{2}\right)_{2}$ with respect to the dimer ac plane is similar to that of $\left(\mathrm{H}_{2} \mathrm{O}\right)_{2},{ }^{17,18}$ that is, one monomer lies in the ac plane while the other monomer has its plane of symmetry perpendicular to the ac plane. The values of $P_{b b}$ for $\mathrm{S}^{18} \mathrm{OO}-\mathrm{OS}^{18} \mathrm{O}, \mathrm{S}^{18} \mathrm{OO}-{ }^{18} \mathrm{OSO}$, and $\left(\mathrm{S}^{18} \mathrm{O}_{2}\right)_{2}$ are $52.70,52.64$, and 55.77 amu $\times \AA^{2}$, respectively. The fact that they are nearly equal to $P_{a a}\left(\mathrm{~S}^{18} \mathrm{O}^{16} \mathrm{O}\right)=52.04 \mathrm{amu} \times \AA^{2}$ and $P_{a u}\left(\mathrm{~S}^{18} \mathrm{O}_{2}\right)=55.18$ amu $\times \AA^{2},{ }^{19}$ respectively, is in agreement with the above structural inferences.

Since $\left(\mathrm{SO}_{2}\right)_{2}$ has a plane of symmetry, the geometry of the complex is determined by three parameters, assuming that the structure of the monomer units is unchanged upon complexation. ${ }^{20}$ The parameters are the center-of-mass distance $R_{c m}$ and the tilt angles, $\theta_{1}$ and $\theta_{2}$, between $R_{c m}$ and the $C_{2}$ axes of the out-of-plane monomer and in-plane monomer, respectively (see Fig. 2). These three parameters can be determined by fitting the moments of inertia of $\left(\mathrm{SO}_{2}\right)_{2}$ and its eight isotopically substituted species. Because only $a$ type transitions were assigned, the $A$ rotational constant is not well determined for several species. Also, the effect of the interconversion motion on $A$ is not known, although this is probably small. Consequently, the moment of inertia, $I_{a}$, was excluded from the fit. The least-squares fit yielded the structural parameters listed in Table $V$ and shown in Fig. 2. The value of $R_{\mathrm{cm}}=3.822$ (1) $\AA$ agrees very well with $R_{\mathrm{cm}}=3.825$ (10) A derived by Nelson et al. ${ }^{1}$ from their normal species data. The shortest distance between atoms of each monomer is the distance $d_{s . o}$ between the sulfur of the out-of-plane subunit and the oxygen from the in-plane subunit. It is $3.21 \AA$ which is approximately equal to the sum of their respective van der Waals radii $\left(r_{s}+r_{o}=3.25 \AA\right) .{ }^{21}$ A simple vector sum of the dipole moments of each mon-

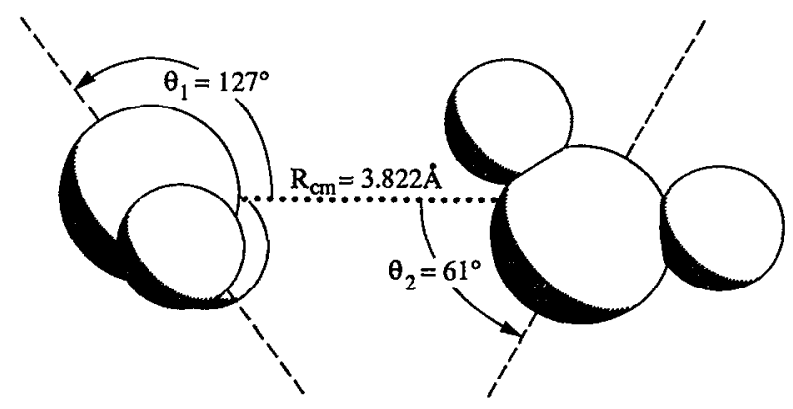

FIG. 2. Molecular structure of $\left(\mathrm{SO}_{2}\right)_{2}$ from the least-squares fit. The plane of the page corresponds to the $a c$ plane of symmetry, where the $a$ axis is nearly collinear to the dashed line $\left(R_{c m}\right)$ joining the centers of mass of each monomer. 
TABLE V. Structural parameters for $\left(\mathrm{SO}_{2}\right)_{2}$ from a least-squares fit of $I_{b}$ and $I$, for all species and from a Kraitchman analysis.

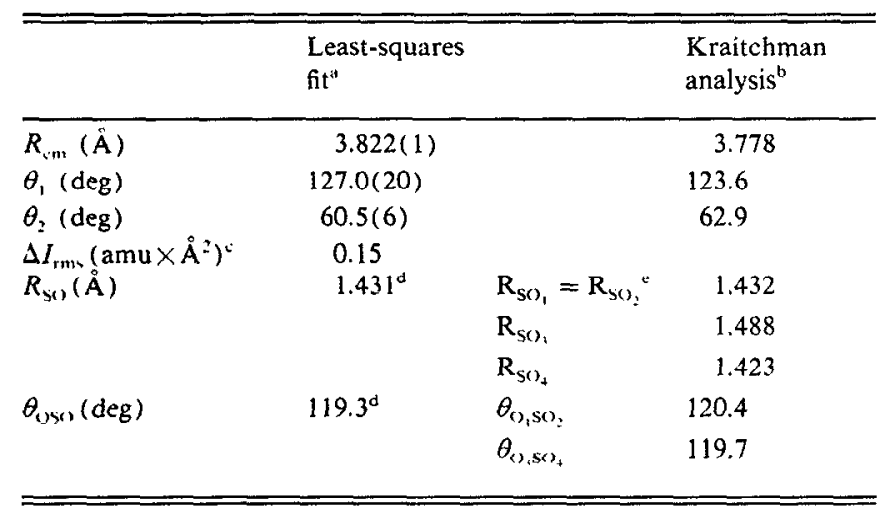

- The uncertainties are $2 \sigma$.

"Reference 22.

$\Delta I=I_{\text {chis }}-I_{\text {isk }}$.

${ }^{J}$ Fixed at the values in $\mathrm{SO}_{2}$ monomer (Ref, 20).

The numbering of oxygens in the dimer: $\mathrm{SO}_{1} \mathrm{O}_{2}-\mathrm{O}_{3} \mathrm{SO}_{4}$.

omer $\left(\mu\left(\mathrm{SO}_{2}\right)=1.63305 \mathrm{D}\right)^{6}$ for this dimer structure yielded $\mu_{a}=1.81 \mathrm{D}$ and $\mu_{c}=0.20 \mathrm{D}$. These values compare well to the observed dipole components for the dimer. An analysis of the least-squares fit showed no other structural orientations which gave a good fit, except for an alternative $\left(\mathrm{H}_{2} \mathrm{O}\right)_{2}$-like structure with $\theta_{1}=88(20)^{\circ}$ and $\theta_{2}=113(2)^{\circ}$. However, the standard deviation of the fit was ten times worse $\left(\Delta I_{\text {rms }}=1.63 \mathrm{amu} \times \AA^{2}\right.$ vs $\left.0.15 \mathrm{amu} \times \AA^{2}\right)$

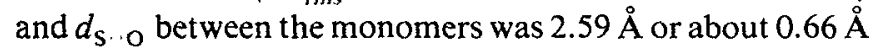
smaller than the sum of the van der Waals radii. When $I_{a}$ was included in the least-squares fit, values of $\theta_{1}=126(9)^{\circ}$, $\theta_{2}=59(2)^{\circ}$, and $R_{\mathrm{cm}}=3.824(2) \AA$ were obtained, which are not greatly different from fitting just the $I_{b}$ and $I_{c}$ 's, however, the deviation of the fit increased to $\Delta I_{\mathrm{rms}}=0.62$ amu $\times \AA^{2}$.

An alternative procedure to derive the structure of the complex employs the Kraitchman method. ${ }^{22}$ Since the rotational constants for five single isotopically substituted species are known, a complete substitution structure $\left(r_{\mathrm{s}}\right.$ structure $)^{2.3}$ can be determined. The results of this analysis are given in Table V. Although the three structural parameters are slightly different compared to the least-squares fit, the orientation of the monomers is basically the same. The structural parameters for each monomer resulting from the Kraitchman analysis are also reported in Table V. The OSO angles of the two subunits are not changed very much. However, the SO bond lengths for the monomer lying in the $a c$ plane are altered considerably. These differences are not believed to be bona fide structural changes, but arise from the fact that vibrationally averaged moments of inertia uncorrected for any tunneling motion are used in the structure calculations.

The precise meaning of the two sets of structural parameters and their relationship to a well-defined set such as the average $\left(r_{z}\right)$ or equilibrium $\left(r_{e}\right)^{23}$ parameters are difficult to determine. This necessitates evaluating the effects of the vibrational motions on the moments of inertia which requires more information about the intermolecular potential than is presently in hand. Since some of the vibrational effects usually cancel in a Kraitchman analysis, this procedure may appear attractive, but the large discrepancy between $d\left(\mathrm{SO}_{3}\right)$ and $d\left(\mathrm{SO}_{4}\right)$ (Table $\mathrm{V}$ ) signals that even this procedure is significantly affected. Consequently, we recommend the use of the least-squares structural parameters with the statistical uncertainties from the fit as an operational structure, i.e., the so-called effective structure $\left(r_{0}\right)$ in the lowest vibrational state. These parameters are probably within $\pm 0.04 \AA$ and $\pm 5^{\circ}$ of the average $\left(r_{z}\right)$ structural parameters by comparison with other systems.

Nelson et al. have previously tried to compare their preliminary structural results for $\left(\mathrm{SO}_{2}\right)_{2}{ }^{1}$ with the structure of two nearest neighbors in the $\mathrm{SO}_{2}$ crystal. ${ }^{24}$ Since more structural information concerning the orientation of each $\mathrm{SO}_{2}$ monomer is available now, it is interesting to make this comparison again. The only similarity between the structure of the $\mathrm{SO}_{2}$ dimer and two nearest neighbors in the $\mathrm{SO}_{2}$ crystal is that in both cases there is a plane of symmetry. However, in the crystal the symmetry axes and dipole moments of the $\mathrm{SO}_{2}$ molecules are almost aligned, i.e., they are nearly parallel rather than nearly antiparallel as observed for the dimer. This suggests that the many-body interaction in the crystal does not allow the structure of the free $\mathrm{SO}_{2}$ dimer to be readily transferred to the crystalline phase. It is interesting that an infrared (IR) study of the $\mathrm{SO}_{2}$ dimer in $\mathrm{N}_{2}$ matrices also indicated a plane of symmetry with nonequivalent $\mathrm{SO}_{2}$ units. ${ }^{25}$ While there was considerable uncertainty about the geometry of the dimer, structures were also suggested in which the dipole moments of each monomer were more nearly aligned.

\section{Electrostatic analysis}

Buckingham and Fowler have proposed a simple physical model that successfully predicts the equilibrium conformation of many van der Waals complexes. ${ }^{26}$ This approach is based on electrostatic interactions between the monomers, where each monomer is described by a set of so-called distributed multipoles. ${ }^{27}$ Furthermore, each atom is described as a hard sphere to account for the short-range repulsion. ${ }^{26}$ Recently, this model was applied to the $\mathrm{SO}_{2}$ complexes with ethylene, acetylene, and $\mathrm{HCN}$. For the $\mathrm{C}_{2} \mathrm{H}_{4}-\mathrm{SO}_{2}$ complex, the equilibrium structure obtained from the electrostatic model was in good agreement with experiment. ${ }^{28}$ For the $\mathrm{HCN}-\mathrm{SO}_{2}$ system, the model predicted a planar $\mathrm{H}$-bonded OSO $\cdots \mathrm{HCN}$ conformation while a $\mathrm{N} \cdots \mathrm{S}$ bonded nonplanar dimer was found experimentally. ${ }^{29,30}$ It was shown that the latter structure would be more stable than the Hbonded form if the hard-sphere radii constraint was relaxcd. In the case of $\mathrm{C}_{2} \mathrm{H}_{2}-\mathrm{SO}_{2}$, the model predicted that a planar $\mathrm{H}$-bonded OSO $\cdots \mathrm{HCCH}$ structure would be more stable than a nearly parallel planes arrangement (like $\mathrm{C}_{2} \mathrm{H}_{4}-\mathrm{SO}_{2}$ ) where the $\pi$ systems of $\mathrm{C}_{2} \mathrm{H}_{2}$ and $\mathrm{SO}_{2}$ are directed at each other. Nevertheless, only the latter arrangement has been observed. ${ }^{31}$ In the present work, we applied this model to $\left(\mathrm{SO}_{2}\right)_{2}$, where the point multipole values for each $\mathrm{SO}_{2}$ subunit were taken from Ref. 26 and the hard sphere van der Waals radii from Ref. 21. Starting from a structure where the molecular plane of one $\mathrm{SO}_{2}$ subunit is perpendicular to 
the molecular plane of the second subunit, the electrostatic energy was calculated for $\phi_{1}$ and $\phi_{2}$ ranging from $-180^{\circ}$ to $180^{\circ}$ at increments of $10^{\circ}$, while the center-of-mass distance $R_{\mathrm{cm}}$ value was controlled by the hard sphere repulsion constraint. The corresponding energy surface is shown as a contour map in Fig. 3. This electrostatic model predicts two different minima. The first minimum at position $A$ $\left(\phi_{1}= \pm 90^{\circ}\right.$ and $\left.\phi_{2}= \pm 70^{\circ}\right)$ has an energy of $-2.8 \mathrm{kcal} /$ mol, whereas the second minimum $B\left(\phi_{1}= \pm 70^{\circ}\right.$ and $\phi_{2}=\mp 40^{\circ}$ ) has a slightly higher energy, $-2.7 \mathrm{kcal} / \mathrm{mol}$. The experimental geometry $(-1.3 \mathrm{kcal} / \mathrm{mol})$ represented by a star $\left(\phi_{1}= \pm 53^{\circ}, \phi_{2}= \pm 119^{\circ}\right)$ in Fig. 3 is closer to the minimum $A$ than to the minimum $B$, although the agreement is not very good for $\phi_{1}$ or $\phi_{2}$.

The experimental structure (and $A$ ) roughly correspond to an arrangement where the $\mathrm{S}-\mathrm{O}$ bond moment of the in-plane monomer is antiparallel to the molecular dipole moment of the out-of-plane monomer. In structure $B$, the SO bond of the in-plane monomer is pointing approximately towards the molecular plane of the other monomer suggestive of a $\mathrm{S} \cdots \mathrm{O}$ interaction and reminiscent of the interaction in the $\mathrm{O}_{2} \mathrm{~S} \cdots \mathrm{NCH}$ species. The prediction of a second isomer is intriguing, although we have no experimental evidence to confirm or disprove its existence. At the present time, we have accounted for virtually all of the transitions which we have observed.

Calculations were also made for the two cases where

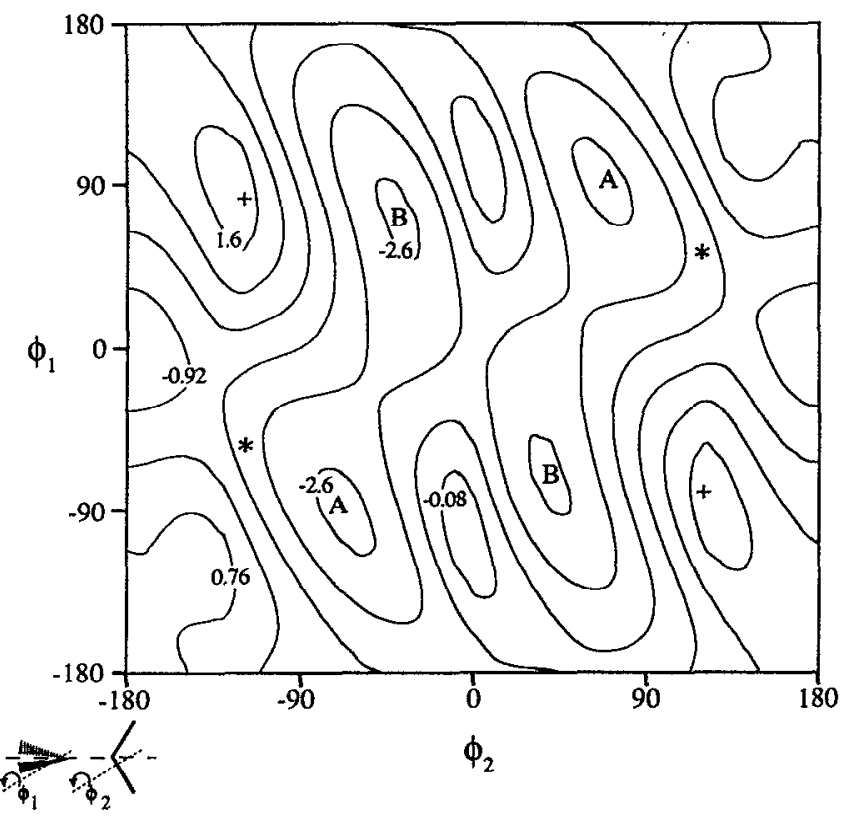

FIG. 3. Electrostatic energy contour map of $\left(\mathrm{SO}_{2}\right)_{2}$, with the molecular symmetry plane of the two subunits perpendicular to each other. The position at $\phi_{1}=-180^{\circ}$ and $\phi_{2}=-180^{\circ}$ corresponds to the structure where the two subunits have their $\mathrm{C}_{2}$ axes collinear and antiparallel with the $S$ atoms closest. The structure at this position is shown at the bottom left of the contour plot. The variation of the two angles $\phi_{1}$ and $\phi_{2}$ from $-180^{\circ}$ to $180^{\circ}$ is performed in a counterclock wise fashion, as shown in the figure. The contour spacing is $0.84 \mathrm{kcal} / \mathrm{mol}$. A and B indicate minima in the potential energy and the " + " corresponds to a maximum. The star indicates the position of the observed structure. All positions at $\phi_{1}$ and $\phi_{2}$ and $-\phi_{1}$ and - , are equivalent by symmetry. both $\mathrm{SO}_{2}$ monomers are always coplanar or where their molecular planes are parallel. For the first case, the lowest energy $(-2.5 \mathrm{kcal} / \mathrm{mol})$ was found for an approximately "parallelogram-like" structure where two SO bonds line up in an antiparallel arrangement. For the second case, where the $\mathrm{SO}_{2}$ molecular planes are parallel, the electrostatic model predicts that this configuration is improbable since the energy was positive for all relative orientations of the respective $C_{2}$ axes of each $\mathrm{SO}_{2}$, even when the planes align with the $\mathrm{SO}_{2}$ dipole moments antiparallel $(+1 \mathrm{kcal} / \mathrm{mol})$.

Furthermore, we carried out electrostatic calculations for $\mathrm{SO}_{2}-\mathrm{H}_{2} \mathrm{O}^{2}$ and $\mathrm{SO}_{2}-\mathrm{H}_{2} \mathrm{~S}^{2,32}$ for which it has been shown experimentally that neither of the subunits is lying in the $a c$ plane of symmetry and that the molecular planes of each subunit are approximately parallel. The distributed multipole values for $\mathrm{H}_{2} \mathrm{O}$ and $\mathrm{H}_{2} \mathrm{~S}$ were used directly from Rer. 26. The electrostatic energy values for four different orientations of these two van der Waals complexes and $\left(\mathrm{SO}_{2}\right)_{2}$ are reported in Table VI. No global minimum search was performed for $\mathrm{H}_{2} \mathrm{O}-\mathrm{SO}_{2}$ and $\mathrm{H}_{2} \mathrm{~S}-\mathrm{SO}_{2}$. In conformations I and II, both subunits planes are perpendicular to the symmetry plane of the complex, whereas in conformations III and IV one of the two subunits is lying in the symmetry plane. The results in Table VI show that $\mathrm{H}_{2} \mathrm{O}-\mathrm{SO}_{2}$ and $\mathrm{H}_{2} \mathrm{~S}-\mathrm{SO}_{2}$, unlike the $\mathrm{SO}_{2}$ dimer, are more stable when their respective subunit planes are nearly parallel to each other. These calculations indicate that the contrast between the observed perpendicular planes structure of the $\mathrm{SO}_{2}$ dimer and the nearly parallel planes structures of $\mathrm{SO}_{2}-\mathrm{H}_{2} \mathrm{O}$ and $\mathrm{SO}_{2}-\mathrm{H}_{2} \mathrm{~S}$ can be rationalized by electrostatic interactions.

\section{SUMMARY}

The assignment of the spectra of the $\left(\mathrm{SO}_{2}\right)_{2}$ isotopic species was necessary to determine the structure and to better understand the interconversion motion. The tunneling analysis applied to $\left(\mathrm{H}_{2} \mathrm{O}\right)_{2}$ by Coudert and Hougen ${ }^{9}$ was found to be very useful in the present case due to the isomorphism in the structures of the two dimers. Since $\mathrm{SO}_{2}$ is much heavier than $\mathrm{H}_{2} \mathrm{O}$ and due to the boson spin statistics in

TABLE VI. Electrostatic energies (in $\mathrm{kcal} / \mathrm{mol}$ ) for different structures of $\mathrm{H}_{2} \mathrm{O}-\mathrm{SO}_{2}, \mathrm{H}_{2} \mathrm{~S}-\mathrm{SO}_{2}$, and $\mathrm{SO}_{2}-\mathrm{SO}_{2}$.

\begin{tabular}{lrrrr}
\hline & $\mathrm{I}^{\mathrm{a}}$ & $\mathrm{II}^{\mathrm{b}}$ & $\mathrm{III}^{\mathrm{c}}$ & $\mathrm{IV}^{\mathrm{d}}$ \\
\hline $\mathrm{H}_{2} \mathrm{O}-\mathrm{SO}_{2}$ & -5.1 & -2.8 & 2.6 & 3.8 \\
$\mathrm{H}_{2} \mathrm{~S}-\mathrm{SO}_{2}$ & -2.3 & -1.6 & 1.5 & 1.8 \\
$\mathrm{SO}_{2}-\mathrm{SO}_{2}$ & 2.8 & 1.0 & -1.3 & $-1.3^{\mathrm{e}}$ \\
\hline
\end{tabular}

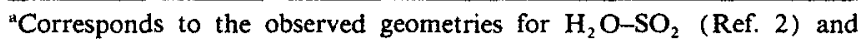
$\mathrm{H}_{2} \mathrm{~S}-\mathrm{SO}_{2}$ (Ref. 2 and 32); for $\mathrm{SO}_{2}-\mathrm{SO}_{2}$ the molecular planes are parallel with the molecular dipoles parallel.

${ }^{b}$ Corresponds to the structure where the subunits have their molecular planes parallel to each other and their dipoles antiparallel.

"Corresponds to structure II followed by a $90^{\circ}$ rotation of $\mathrm{SO}_{2}$ about its $C_{2}$ axis; for $\mathrm{SO}_{2}-\mathrm{SO}_{2}$ this corresponds to the observed geometry.

${ }^{d}$ Corresponds to the structure II followed by a $90^{\circ}$ rotation of $\mathrm{H}_{2} \mathrm{O}$ (or $\mathrm{H}_{2} \mathrm{~S}$ ) about its $C_{2}$ axis.

eSame conformation as III for $\mathrm{SO}_{2}-\mathrm{SO}_{2}$. 
$\left(\mathrm{SO}_{2}\right)_{2}$ rather than fermion, the spectra of $\left(\mathrm{SO}_{2}\right)_{2}$ were less complicated than in the water dimer. Only one internal motion could be detected and a simpler Hamiltonian could be applied to fit the spectra of $\left(\mathrm{SO}_{2}\right)_{2}$. This is the $1 \rightarrow 5$ interconversion and $h_{5,}$ is $-0.017 \mathrm{MHz}$ for $\left(\mathrm{SO}_{2}\right)_{2}$ compared to $-5261 \mathrm{MHz}$ for $\left(\mathrm{H}_{2} \mathrm{O}\right)_{2} .^{9(\mathrm{~b})}$ The tunneling splitting from the $1 \rightarrow 4$ motion was not observed for $\mathrm{S}^{18} \mathrm{OO}$-OSO unlike the water dimer where it produces the largest effect $\left(h_{4 r}=-47353 \mathrm{MHz}\right)$. This is not surprising given the differences in positions (and masses) of the hydrogens and oxygens which tunnel in $\left(\mathrm{H}_{2} \mathrm{O}\right)_{2}$ and $\left(\mathrm{SO}_{2}\right)_{2}$. Rough calculations using an approximately correct internal rotation Hamiltonian to model the internal rotation of the out-ofplane $\mathrm{SO}_{2}$ about its $C_{2}$ axis, indicates that this barrier must be below $35 \mathrm{~cm}^{-1}$ to produce observable splittings, setting a lower limit for the barrier.

In $\left(\mathrm{H}_{2} \mathrm{O}\right)_{2}, h_{2 v}$ and $h_{7 v}$ are -746 and $-378 \mathrm{MHz}$, respectively. The absence of splitting in the $\mathrm{S}^{18} \mathrm{OO}-\mathrm{OSO}$ species indicates that the $1 \rightarrow 2$ motion does not affect the spectrum. The absence of further perturbations in the spectra from species such as $\left(\mathrm{S}^{16} \mathrm{O}_{2}\right)_{2},\left(\mathrm{~S}^{18} \mathrm{O}_{2}\right)_{2}, \mathrm{~S}^{18} \mathrm{OO}-{ }^{18} \mathrm{OSO}$, and $\mathrm{S}^{18} \mathrm{OO}-\mathrm{OS}^{18} \mathrm{O}$ indicates that the $1 \rightarrow 7$ interchange is quenched, a result consistent with simple steric arguments and the absence of the $1 \rightarrow 4$ tunneling motion, where similar steric interactions also occur. The only tunneling motion for which the spectroscopy is effectively silent is the $1 \rightarrow 3$ tunneling motion. This motion also cannot be eliminated on simple steric arguments.

Electrostatic calculations were also helpful, to some extent, in rationalizing the structure of the $\mathrm{SO}_{2}$ dimer. These calculations when carried out for $\left(\mathrm{SO}_{2}\right)_{2}, \mathrm{H}_{2} \mathrm{O}-\mathrm{SO}_{2}$, and $\mathrm{H}_{2} \mathrm{~S}_{-} \mathrm{SO}_{2}$ showed that the orientation of the subunits with respect to the plane of symmetry of the complex is apparently of electrostatic origin. On the other hand, the Buckingham and Fowler model was not very reliable in predicting the observed tilt angles of each $\mathrm{SO}_{2}$ monomer in $\left(\mathrm{SO}_{2}\right)_{2}$. It also predicts that at least two other structures have low energies which could make them observable. The validity of these latter two predictions cannot be evaluated at this time. Finally, the electrostatic model predicts a binding energy of $1.3 \mathrm{kcal} / \mathrm{mol}$ for the experimental geometry which can be compared to the value of 0.8 obtained from $D_{J}$ and the pseudodiatomic approximation. ${ }^{33}$ We conclude that the electrostatic model applied to $\left(\mathrm{SO}_{2}\right)_{2}$ is partially successful in interpreting the geometric results and bonding energetics but probably in need of a more sophisticated parameterization of the repulsive term and perhaps addition of other terms like polarization and dispersion in order to more reliably describe this system. Nevertheless, in its present simple form, it provides a useful starting point for a semiquantitative analysis of the experimental observations.

\section{ACKNOWLEDGMENTS}

Helpful discussions with Dr. L. H. Coudert are acknowledged. This work has been supported by a grant from the National Science Foundation (CHEM-8917945). Acknowledgment is also made to the Donors of the Petroleum
Research Fund, administered by the American Chemical Society for the partial support of this work (ACS-PRF \#21061-AC6).

' D. D. Nelson, Jr., G. T. Fraser, and W. Klemperer, J. Chem. Phys. 83, 945 (1985).

${ }^{2}$ K. Matsumura, F. J. Lovas, and R. D. Suenram, J. Chem. Phys. 91, 5887 (1989).

${ }^{3}$ (a) M. S. LaBarge, J. J. Oh, K. W. Hillig II, and R. L. Kuczkowski, Chem. Phys. Lett. 159, 559 (1989), (b) A. Taleb-Bendiab, K. W. Hillig II, and R. L. Kuczkowski (in preparation).

${ }^{4}$ K. W. Hillig II, J. Matos, A. Scioly, and R. L. Kuczkowski, Chem. Phys. Lett. 133, 359 (1987).

${ }^{5}$ R. K. Bohn, K. W. Hillig II, and R. L. Kuczkowski, J. Phys. Chem. 93, 3456 (1989).

${ }^{6}$ D. Patel, D. Margolese, and T. R. Dyke, J. Chem. Phys. 70, 2740 (1979).

${ }^{7}$ T. R. Dyke, J. Chem. Phys. 66, 492 (1977).

${ }^{8}$ J. T. Hougen, J. Mol. Spectrosc. 114, 395 (1985).

${ }^{9}$ (a) L. H. Coudert and J. T. Hougen, J. Mol. Spectrosc. 130, 86 (1988). (b) 139, 259 (1990).

${ }^{10}$ T. R. Dyke, B. J. Howard, and W. Klemperer, J. Chem. Phys. 56, 2442 (1972).

" J. K. G. Watson, Vibrational Spectra and Structure, edited by J. R. Durig (Elsevier, Amsterdam 1977), Vol 6, Chap. 1.

${ }^{12}$ See AIP document No. PAPS JCPSA-94-6956-9 for 9 pages of tables of transitions for the isotopes of $\left(\mathrm{SO}_{2}\right)_{2}$. Order by PAPS number and journal reference from American Institute of Physics, Physics Auxiliary Publication Service, 335 East 45th Street, New York, NY 10017. The price is $\$ 1.50$ for each microfiche ( 98 pages) or $\$ 5.00$ for photocopies of up to 30 pages, and $\$ 0.15$ for each additional page over 30 pages. Airmail additional. Make checks payable to the American Institute of Physics.

${ }^{13}$ N. Ohashi and A. S. Pine, J. Chem. Phys. 81, 73 (1984).

${ }^{14}$ L. H. Coudert, F. J. Lovas, R. D. Suenram, and J. T. Hougen, J. Chem. Phys. 87, 6290 (1987).

${ }^{15}$ P. A. Helminger and F. C. DeLucia, J. Mol. Spectrosc. 111, 66 (1985).

${ }^{16}$ R. van Riet, G. Steenbeckeliers, Ann. Soc. Sci. Bruxelles, Ser. I, 97, 117 (1983).

${ }^{17}$ T. R. Dyke, K. M. Mach, and J. S. Muenter, J. Chem. Phys. 66, 498 (1977).

${ }^{18}$ J. A. Odutola and T. R. Dyke, J. Chem. Phys. 72, 5062 (1980).

${ }^{19} \mathrm{~J}$. Lindenmayer, H. Jones, and V. Typke, J. Mol. Spectrosc. 110, 357 (1985).

${ }^{20}$ M. D. Harmony, V. W. Laurie, R. L. Kuczkowski, R. H. Schwendeman, D. A. Ramsey, F. J. Lovas, W. J. Lafferty, and A. G. Maki, J. Phys. Chem. Ref. Data 8, 619 (1979).

${ }^{21}$ L. Pauling, The Nature of the Chemical Bond (Cornell Univ., Ithaca, New York, 1960) p. 260.

${ }^{22}$ J. Kraitchman, Am. J. Phys. 21, 17 (1953).

${ }^{23}$ R. Schwendeman in Critical Evaluation of Chemical and Physical Structural Information, edited by D. R. Lide and M. A. Paul (National Academy of Sciences, Washington D.C., 1974), pp. 94-115.

${ }^{24}$ B. Post, R. S. Schwartz, and I. Frankuchen, Acta Crystallogr. 5, 372 (1952).

${ }^{25}$ L. Nord, J. Mol. Struct. 96, 19 (1982).

${ }^{26}$ A. D. Buckingham and P. W. Fowler, Can. J. Chem. 63, 2018 (1985).

${ }^{27}$ A. J. Stone, Chem. Phys. Lett. 83, 233 (1981).

${ }^{28}$ A. M. Andrews, A. Taleb-Bendiab, M. S. LaBarge, K. W. Hillig II, and R. L. Kuczkowski, J. Chem. Phys. 93, 7030 (1990).

${ }^{29}$ E. J. Goodwin and A. C. Legon, J. Chem. Phys. 85, 6828 (1986).

${ }^{30}$ A. C. Legon, Structure and Dynamics of Weakly Bound Molecular Complexes, edited by A. Weber (D. Reidel, Dordrecht, Holland, 1987), p. 23.

${ }^{31}$ A. M. Andrews, K. W. Hillig II, R. L. Kuczkowski, A. C. Legon, and N. Howard, J. Chem. Phys. 94, 6947 (1991).

${ }^{32}$ (a) R. E. Bumgarner, D. J. Pauley, and S. G. Kukolich, J. Chem. Phys. 87, 3749 (1987). (b) D. J. Pauley and S. G. Kukolich, ibid. 93, 1487 (1990).

${ }^{33}$ D. J. Millen, Can. J. Chem., 63, 1477 (1985). Equation (20) of this reference was used to calculate the stretching force constant. 